\title{
La naissance de Sparte : entre sources littéraires et sources archéologiques
}

The Formation of Sparta: Between Literary Sources and Archaeological Evidence

\section{Valeria Tosti}

\section{(2) OpenEdition}

1 Journals

Édition électronique

URL : https://journals.openedition.org/gaia/839

DOI : $10.4000 /$ gaia.839

ISSN : 2275-4776

Éditeur

UGA Éditions/Université Grenoble Alpes

\section{Édition imprimée}

ISBN : 978-2-37747-199-7

ISSN : 1287-3349

\section{Référence électronique}

Valeria Tosti, «La naissance de Sparte : entre sources littéraires et sources archéologiques », Gaia [En ligne], 22-23 | 2020, mis en ligne le 30 juin 2020, consulté le 09 décembre 2021. URL : http://

journals.openedition.org/gaia/839; DOI : https://doi.org/10.4000/gaia.839

Ce document a été généré automatiquement le 9 décembre 2021.

Gaia. Revue interdisciplinaire sur la Grèce archaïque 


\title{
La naissance de Sparte : entre sources littéraires et sources archéologiques
}

The Formation of Sparta: Between Literary Sources and Archaeological Evidence

\author{
Valeria Tosti
}

\section{Introduction}

1 Bien que Sparte, considérée du point de vue politique, idéologique et social comme unique, ait toujours suscité un grand intérêt, la recherche n'a toujours pas réussi à reconstituer l'image complète de sa forme et de sa structure. En général, l'attention des études sur les origines de la cité et la naissance des institutions politiques est focalisée sur la figure légendaire de Lycurgue, ainsi que sur l'arrivée des Doriens dans le Péloponnèse, à la suite de l'effondrement des palais mycéniens à la fin du $\mathrm{II}^{\mathrm{e}}$ millénaire av. J.-C. Par conséquence, les données qui nous sont aujourd'hui parvenues concernant la polis des Lacédémoniens sont le résultat d'un passé déformé par des stéréotypes sclérosés autour de transmissions littéraires mal interprétées et par un paysage archéologique désolant, dont la lecture a créé un modèle tant idéalisé qu'irréaliste. En dépit des difficultés objectives qui peuvent entraver la compréhension et la définition des phénomènes de la naissance et du développement de Sparte, il semble nécessaire de réexaminer cette question, tout d'abord en montrant les limites des méthodes adoptées jusqu'ici ainsi que les lacunes dans la documentation spartiate.

\section{Histoire de la recherche}

2 Le célèbre passage de Thucydide ${ }^{1}$, où l'historien décrit le mode d'habitat de Sparte en kata kômas, selon l'ancienne manière (archaios tropos) de l'Hellade, a influencé les savants depuis la redécouverte de la Grèce, à tel point que le roi Othon et ses 
architectes furent amenés à construire la nouvelle Sparte en 1834 exactement à l'emplacement de l'ancienne, en faisant une " cité de l'ouest » et annihilant ainsi toute trace d'un passé «embarrassant ${ }^{2}$ » à cause de la monumentalité insuffisante et de l'urbanisation à "l'ancienne ». La superposition complète de la ville moderne sur l'ancienne a considérablement aggravé la difficulté de repérer les traces antiques qui sont déjà assez rares sur le terrain. L'importance attribuée au texte de Thucydide $(\mathrm{I}, 10,2)$ a également découragé les recherches archéologiques, guère motivées par la découverte d'une réalité peu monumentale.

3 Ensuite, le climat historique et culturel des États-Unis dans la première moitié du $\mathrm{xx}^{\mathrm{e}}$ siècle conduisit à une interprétation de l'histoire ancienne de Sparte dans une perspective nazie, ce qui a conduit les archéologues américains à choisir de fouiller plutôt l'agora d'Athènes, berceau de la démocratie, forme de gouvernement dont les États-Unis prétendaient être les héritiers directs³.

4 Notre connaissance archéologique de la ville est due pour une bonne partie aux fouilles conduites par la British School at Athens au début du $\mathrm{xx}^{\mathrm{e}}$ siècle dans la région de l'Acropole et dans le sanctuaire d'Artémis Orthia ${ }^{4}$. Les recherches se sont ensuite limitées à des fouilles de sauvetage menées par la $5^{2}$ Éphorie des Antiquités préhistoriques et classiques, qui ont mis au jour des morceaux de la ville antique et dont les résultats sont en partie publiés dans l'Archaiologikon Deltion ${ }^{5}$.

5 Enfin, l'étude de l'ancienne polis de Sparte a été fortement conditionnée par la création de modèles qui ont peu à voir avec la réalité de la période de la formation de la polis et des époques archaïque et classique. Il s'agit tout d'abord d'un modèle socio-culturel dont les effets ont longtemps été reconnus et définis comme "mirage spartiate ${ }^{6} »:$ un phénomène d'idéalisation de l'histoire et de la société lacédémonienne produit dès le $\mathrm{v}^{\mathrm{e}}$ siècle av. J.-C. par des groupes oligarchiques athéniens ${ }^{7}$. Par ailleurs, selon une tradition du XVIII ${ }^{\mathrm{e}}$ siècle ${ }^{8}$, reprise par L. Pareti en 1910 et de manière plus cohérente par H. T. Wade-Gery ${ }^{9}$, la Sparte kata kômas de Thucydide correspondrait à cinq villages étendus sans interruption topographique - à l'exception d'Amyclées - à l'intérieur de l'enceinte hellénistique, puisque cinq était le nombre des éphores. Cette théorie a reçu un tel consensus que presque toutes les études sur la topographie et l'archéologie de Sparte se sont appuyées sur ce modèle des quatre villages contigus, ensemble délimité par les murs de fortification de la ville hellénistico-romaine, et d'un cinquième village, à $5 \mathrm{~km}$ de la ville, situé sur la colline de Agia Kyriaki et correspondant au sanctuaire d'Apollon Amycléen.

6 Il en résulte la création d'un modèle rigide, fondé sur l'interprétation des sources littéraires et épigraphiques postérieures à la polis kata kômas décrite par Thucydide ${ }^{10}$ : ce modèle, accepté de manière conventionnelle et encore aujourd'hui dominant, a été mis en discussion d'une manière tout à fait convaincante par Marcello Lupi ${ }^{11}$ : selon l'historien italien, le nom et le nombre de villages ne sont pas attestés par la documentation contemporaine de la polis kata kômas des époques archaïque et classique, mais par des inscriptions et des sources postérieures ${ }^{12}$. L'attribution à l'époque archaïque des quatre villages mentionnés par Pausanias (Pitanè, Limnai, Kynosoura, Mesoa) est abusive. La position centrale de Pitanè suggérée par les sources antiques dépend du fait qu'elle représente le vrai centre urbain de Sparte dans une cité composée de plusieurs villages. 


\section{Avant la naissance de la ville de Sparte : l'âge du bronze}

7 Située le long des rives occidentales du fleuve Eurotas, qui se jette dans la mer à environ $40 \mathrm{~km}$ plus au sud, Sparte domine une plaine alluviale fertile cernée par deux chaînes de montagnes : le Taygète à l'ouest, qui a l'aspect d'une barrière culminant à $2404 \mathrm{~m}$, et le Parnon à l'est, ne dépassant pas les $1935 \mathrm{~m}$. Située entre ces deux massifs montagneux et bordée au sud par une bande vallonnée la séparant de la mer, la plaine de Sparte justifie l'épithète homérique de « creuse Lacédémone » (koilè Lakedaimon) ${ }^{13}$.

8 L'absence, en Laconie, d'un centre palatial comparable à ceux de l'Argolide, de la Béotie et de la Messénie, et d'archives des tablettes en linéaire $\mathrm{B}$ a signifié que la région a longtemps été reléguée à un rôle de deuxième niveau dans le débat scientifique sur la géographie politique et territoriale des États mycéniens ${ }^{14}$. Pourtant, dans le passage iliadique du " catalogue des vaisseaux ", résultant de l'adaptation au viII siècle av. J.-C. d'une liste ancienne, sont répertoriés les contingents achéens qui ont participé à l'expédition de Troie, y compris sur le territoire de la "creuse Lacédémone ", qui inclut, outre Sparte, d'autres villes dans la plaine environnante ${ }^{15}$. On a retrouvé également à Thèbes trois tablettes écrites en linéaire B et datées de la seconde moitié du XII siècle av. J.-C. mentionnant des donateurs d'offrandes dont un Lakédaimon et son fils (TH Gp 227 : ra-ke-da-mo-ni-jo-u-jo, fils de Lakedaimonios) ${ }^{16}$. Il n'est pas certain que "Lakédaimon" soit l'ethnonyme désignant un habitant de Lacédémon ou un anthroponyme, mais, en tout cas, sa présence dans les tablettes thébaines présuppose déjà l'existence à l'époque mycénienne d'une localité appelée Lacédémone, avec laquelle le palais de Thèbes a maintenu des rapports ${ }^{17}$.

9 En outre, les premiers témoignages littéraires nous transmettent l'image d'une Sparte bien construite, composée de grands espaces ouverts avec de larges rues, une ville digne d'être vue, qui suscitait l'émerveillement des poètes et des ambassadeurs qui la

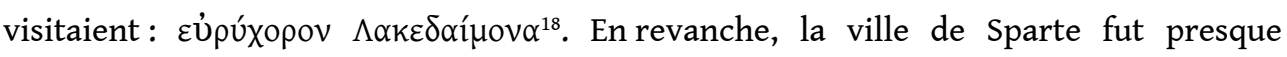
inhabitée à l'âge du bronze et avait certainement un rôle marginal par rapport à d'autres centres prospères de la vallée de l'Eurotas, identifiés avec le "palais de Ménélas » (fig. 1). 
Fig. 1. - Carte la vallée de l'Eurotas avec les principaux sites de la fin de l'âge du bronze (d'après Hope Simpson, 2009, 317, fig. 1).

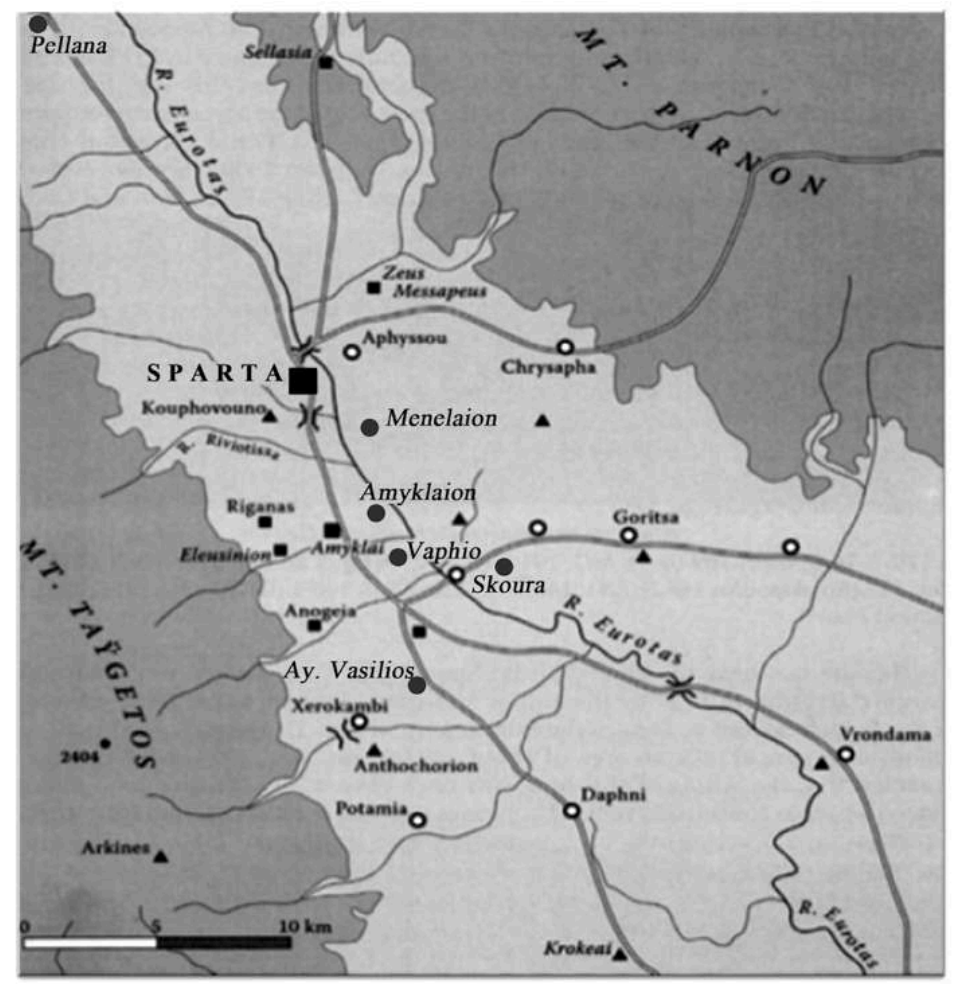

\section{a. Sparte}

Des fragments sporadiques de céramique datés du HM et du HR ont été trouvés sur l'Acropole de Sparte et les zones environnantes ${ }^{19}$. Dans la région de Psychiko ${ }^{20}$, sept tombes (HM-HR) ont été découvertes, tandis qu'il ne reste aucune trace des maisons correspondantes ${ }^{21}$. Un autre habitat a été identifié entre le bord sud-ouest de la ville et la localité voisine d'Agia Irini ${ }^{22}$.

\section{b. Therapne}

11 Des découvertes bien plus importantes ont été faites dans les secteurs entourant la zone de la future polis. À quelques kilomètres de Sparte, sur la crête de Therapne, sur la rive orientale de l'Eurotas, a été déterré l'un des plus importants sites mycéniens de la Laconie, qui a fait l'objet d'enquêtes systématiques par la British School at Athens en 1909-1910 et 1973-1985²3. Sur chacune des trois collines, North Hill, Ménélaion et Aétos, la céramique témoigne de l'occupation des sites du HM à l'HR III C. Dans la zone NE de la colline du Ménélaion, à $150 \mathrm{~m}$ à l'est du sanctuaire archaïque de Ménélas et d'Hélène, les fouilles ont identifié un complexe monumental, dont les phases d'occupation s'étendent du HR IIB ${ }^{24}$ au HR IIIA1 ${ }^{25}$, jusqu'au HR IIIA2-HR IIIB ${ }^{26}$, avec une réoccupation partielle au début du HR IIIC ${ }^{27}$.

\section{c. Agia Kiriaki}

De la colline d'Agia Kiriaki, le site du futur sanctuaire d'Apollon Amycléen, proviennent des objets votifs ${ }^{28}$, comme par exemple 136 figurines zoomorphes en terre cuite et 
d'autres du type $\Psi$ datées du HR IIIB-HR IIIC final, avec quelques fragments de céramique contemporaine, attestant l'existence d'un culte mycénien sur la colline. Aucune structure de maçonnerie n'a été trouvée, soit parce que le sanctuaire archaïque a remplacé des structures plus anciennes, soit parce qu'un péribole simple entourait un culte en plein air. La dispersion et l'état fragmentaire du matériel, trouvé dans des couches perturbées en association avec des matériels de la période protogéométrique et géométrique, pourraient renforcer la première hypothèse ${ }^{29}$. P. Calligas défend une opinion différente et explique la provenance du matériel mycénien à partir d'un remblai de terre qui aurait été prélevé sur un site voisin et ensuite introduit dans la zone pour des travaux de terrassement subséquents ${ }^{30}$. L'analyse du matériel et les études les plus récentes ont établi le début du culte dans le HR IIIB2 (seconde moitié XIII ${ }^{\mathrm{e}}$ siècle av. J.-C.) ; il a continué à prospérer pendant la période post-palatiale pendant tout le HR IIIC et le submycénien, jusqu'à la deuxième moitié du XI siècle av. J.-C.. ${ }^{31}$. La preuve d'une activité religieuse intense est confirmée par le nombre et la qualité du matériel, y compris deux pièces extraordinaires, une tête de femme avec le polos, presque grandeur nature, et une main tenant une kylix, toutes les deux datées de HR IIIB (XII siècle av. J.-C.).

13 L'habitat de référence n'a pas encore été identifié et ne peut pas correspondre aux structures creusées le long des pentes sud-ouest de cette même colline, qui datent d'une phase immédiatement précédente la naissance du culte (HR IIIA-IIIB), ni aux six tombes à chambre ellipsoïdale ou irrégulièrement quadrangulaire, trouvées à Spilakia et contenant de riches mobiliers funéraires (HR IIIA-IIB ; une tombe du HR IIIC) ${ }^{32}$. Peutêtre qu'il pourrait être identifié dans la zone du village moderne, à l'ouest de la colline.

\section{d. Pellana, Melathria, Vaphio, Palaiopyrgi}

14 Dans la région au nord de Sparte, presque à la frontière avec l'Arcadie, sur le site de Palaiokastro, identifié avec l'ancienne Pellana, il y a des signes de croissance pendant le HR II, comme l'indiquent plusieurs tombes monumentales et une tombe à tholos, ainsi que les vestiges de structures architecturales ${ }^{33}$. Six tombes à chambre creusées dans la roche ont été découvertes en 1966 à Melathria (Skoura), à quelques kilomètres de Sparte et à l'est de Vaphio. Toutes les tombes, sauf une, avaient été pillées. La céramique date du HR IIIA1-HR IIIA2 $2^{34}$. À 8-9 km au sud de Sparte, se trouve la tholos monumentale de Vaphio du HR IIA (1500 av.J.-C.), fouillée par Christos Tsountas en 1889. Bien que pillée déjà dans l'Antiquité, elle conservait encore un mobilier extraordinaire, parmi lequel les fameux gobelets en or décorés en relief et aujourd'hui conservés au Musée archéologique national d'Athènes ${ }^{35}$.

15 L'habitat correspondant doit être cherché dans les environs de Palaiopyrgi, où les fouilles ont révélé l'existence de murs, mal conservés, ainsi que de la céramique attestant une continuité d'occupation dans ce secteur, de la fin du HM au HR IIIA-B ${ }^{36}$. La richesse et la monumentalité de la tombe suggéreraient l'existence d'une élite dans le HR IIA, mais qui a probablement vécu à Paloiopyrgi déjà au $\mathrm{HM}^{37}$.

\section{e. Agios Vasilios}

L'habitat de Agios Vasilios dans la localité de Xirokambi, à environ $12 \mathrm{~km}$ au sud de la ville de Sparte, découvert en $1956^{38}$, a été, à partir de 2010, l'objet de fouilles 
systématiques après la découverte accidentelle en 2008 des trois premières tablettes en linéaire B de la Laconie ${ }^{39}$ (fig. 2), ainsi que de fragments de peintures murales.

Fig. 2. - À gauche, fragments de peintures murales (d'après Vlachopoulos, 2012) ; à droite, tablette en linéaire B (d'après Aravantinos \& Vasilogamvrou, 2012) du site d'Haghios Vasilios.
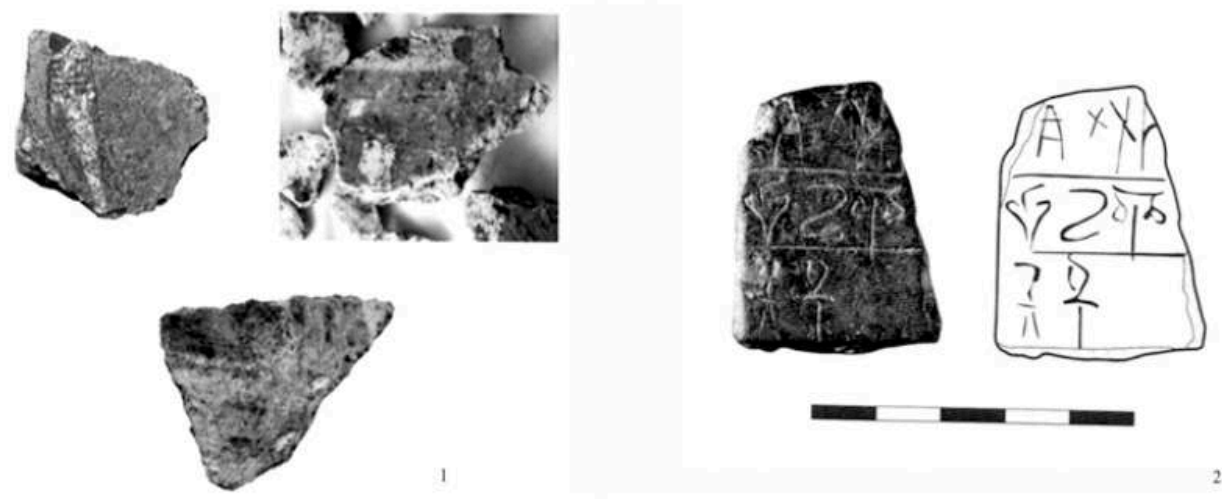

Les fouilles sont en train de mettre au jour un habitat de taille considérable, dont la phase principale est datée des XIV $\mathrm{X}^{\mathrm{e}} \mathrm{XIII}{ }^{\mathrm{e}}$ siècles av. J.-C..$^{40}$. Cette découverte ainsi que les premiers documents en linéaire $B$ de la Laconie révèlent une certaine expérience bureaucratique sous le contrôle d'un pouvoir: il est désormais possible d'imaginer - non sans une certaine hésitation - que ce site fut un important centre politique et administratif de l'époque mycénienne.

\section{4. Âge du fer et l'émergence de la ville dorienne}

Comme dans le reste de la Grèce, la Laconie entre le XIII ${ }^{e}$ et le XII ${ }^{e}$ siècle vit une période de crise et d'instabilitét ${ }^{41}$. C'est le moment où les centres de pouvoir palatiaux n'existent plus, mais où la polis n'est pas encore émergée. Le site du Ménélaion, qui est le plus connu archéologiquement, est abandonné vers 1200 av. J.-C. ; le palais d'Agios Vasilios est incendié pendant la même période. Un appauvrissement général et la dépopulation de la région, qui durera jusqu'au viII ${ }^{\mathrm{e}}$ siècle av. J.-C., est déductible de la rareté des attestations matérielles et de la survie de seulement quelques-uns des nombreux sites mycéniens au XII ${ }^{\mathrm{e}}$ siècle. Ce nombre diminue davantage au XI ${ }^{\mathrm{e}}$ siècle av. J.-C. ${ }^{42}$.

Le seul site qui survit à l'âge du fer est la colline d'Agia Kyriaki, qui devient le nœud de la région. Le matériel provient de contextes stratigraphiques bouleversés sur le plateau de la colline. La céramique, datée du protogéométrique laconien ${ }^{43}$, provient des couches de HR IIIC. Sur cette base, William Coulson constate plutôt une rupture d'occupation ${ }^{44}$. La quantité de céramique protogéométrique retrouvée à Sparte, surtout dans les principaux lieux de culte qui commencent à se développer à cette époque ${ }^{45}$, est bien inférieure à celle trouvée dans le sanctuaire d'Apollon Amycléen. Cela peut servir de preuve du fait que, dans cette phase transitoire, Sparte doit encore être considérée comme périphérique, tandis que le sanctuaire d'Amyclées était le principal lieu de culte de la plaine spartiate pendant cette période ${ }^{46}$.

La nette discontinuité spatiale qui est attestée pendant l'âge du fer dans la vallée de l'Eurotas est le témoignage le plus fiable d'un changement sans équivoque, qui trouvera son sens complet dans la phase suivante. Le moment de l'abandon des sites, qui à l'âge du bronze tardif avait caractérisé l'histoire économique et politique de la Laconie, 
correspond au début d'une occupation intentionnelle du site de la future ville de Sparte. Ce site, comme nous l'avons vu, était presque inhabité précédemment. Le secteur de la future Acropole et ceux adjacents commencent à être occupés en permanence. De récentes découvertes montrent une occupation du site déjà au $\mathrm{XI}^{\mathrm{e}}$ siècle ${ }^{47}$, pendant la période protogéométrique (1050-900 av.J.-C.), avec un développement significatif à l'époque géométrique. En Laconie, la nette discontinuité spatiale dans les $\mathrm{XI}^{\mathrm{e}}$ et $\mathrm{X}^{\mathrm{e}}$ siècles av. J.-C. correspond à la fondation et à la naissance de Sparte ${ }^{48}$. En soi, la discontinuité d'occupation du territoire ne dit rien sur la tradition de l'arrivée des Doriens, et on ne veut pas identifier mécaniquement les fondateurs de Sparte avec ce peuple du nord ${ }^{49}$. Aborder le sujet épineux de l'invasion dorienne est une tâche courageuse qui amène à des problématiques historiographiques divergentes ${ }^{50}$. Nous ne pouvons en aucun cas ignorer la coïncidence significative traçable en Laconie à ce moment de l'histoire : la fin du monde chanté par l'épopée homérique correspond à un événement migratoire transmis par l'ancienne tradition, l'arrivée des Doriens.

21 L'autre discontinuité est de nature linguistique : la différence devient évidente entre le dialecte dorien, avec des traits particulièrement conservateurs, parlés dans la Laconie du $\mathrm{I}^{\mathrm{er}}$ millénaire av. J.-C. et la langue reconnaissable dans les tablettes en linéaire B. Cette discontinuité témoignerait de l'arrivée d'une nouvelle population du nord, qui parlait un dialecte différent de celui des gens qui peuplaient la région laconienne à l'âge du bronze tardif ${ }^{51}$.

Mais le contexte est beaucoup plus complexe. Dans une Laconie peu habitée et en pleine crise après la chute des palais mycéniens, des populations provenant du nord sont arrivées dans des conditions inconnues. Elles ont progressivement fusionné avec la faible population préexistante et ont fondé un nouveau centre qui deviendra la polis de Sparte. Il ne s'agissait pas d'une invasion associée à l'expulsion des peuples autochtones, ni de leur asservissement par un peuple qui se réclamait depuis le début d'une identité dorienne. La revendication des Spartiates de leur origine dorienne est le résultat de leur construction identitaire attestée depuis l'époque archaïque ${ }^{52}$. Pausanias (III, 2, 6) affirme que les Doriens, immédiatement après la fondation de Sparte, auraient été en conflit avec Amyclées, la dernière communauté achéenne existante; ils l'auraient conquise et l'auraient incluse dans la nouvelle ville dorienne. La documentation céramique d'Amyclées ne montre par ailleurs aucune différence de style et de formes avec les céramiques spartiates contemporaines. Selon Marcello Lupi, il est probable que le village d'Amyclées ait fait partie depuis le début de la communauté des Spartiates, contrairement à l'idée qui en faisait le dernier rempart achéen conquis par les envahisseurs Doriens $s^{53}$. Plus que dans les sources littéraires, les traces du processus de formation de cette nouvelle communauté doivent être recherchées dans les témoignages archéologiques, en particulier dans la fondation des lieux de culte.

\section{Les realia spartiates à l'âge du fer}

23 À l'époque protogéométrique et géométrique on constate, contrairement à la période précédente, une forte concentration de trouvailles dans la ville de Sparte, en particulier à l'est de l'Acropole, avec une quantité significative de céramique et des tombes, à côté desquelles se développent des lieux de culte, identifiés par leurs dépôts votifs. Il est possible d'entrevoir une articulation de l'espace, qui est décrit par Thucydide comme 
kata kômas, c'est-à-dire par villages, et qui est plus génériquement le premier mode d'occupation des poleis grecques.

Il est nécessaire de mentionner (fig. 3), dans la rue Staufert 5 (îlot 97), la découverte de cinq tombes à fosse, très proches les unes des autres, datées entre la fin de la période protogéométrique et le début de la période géométrique (fig. 4) (54 $^{54} 150 \mathrm{~m}$ au nord nord-est de cette petite nécropole a été trouvée une autre tombe masculine à l'intérieur d'un pithos, caractérisé par la présence, en plus des os, d'un mobilier particulièrement riche $^{55}$. À $50 \mathrm{~m}$ de distance, un lieu de culte héroïque fut établi à l'époque archaïque sur une tombe de la période géométrique (fig.5 $)^{56}$. Au sud de la zone funéraire fut découvert un édifice octogonal dont les fondations reposent sur une couche riche en céramiques de la période géométrique tardive et du début de la période archaïque ${ }^{57}$. Dans l'îlot 102, un riche dépôt votif comportait du matériel datant de la période géométrique à la période romaine ${ }^{58}$.

Fig. 3. - Carte de Sparte moderne avec la division en îlots et les vestiges monumentaux sur l'Acropole et les lieux principaux d'occupation en époque protogéométrique et géométrique (d'après Zavvou \& Themos, 2009, fig. 11.2).

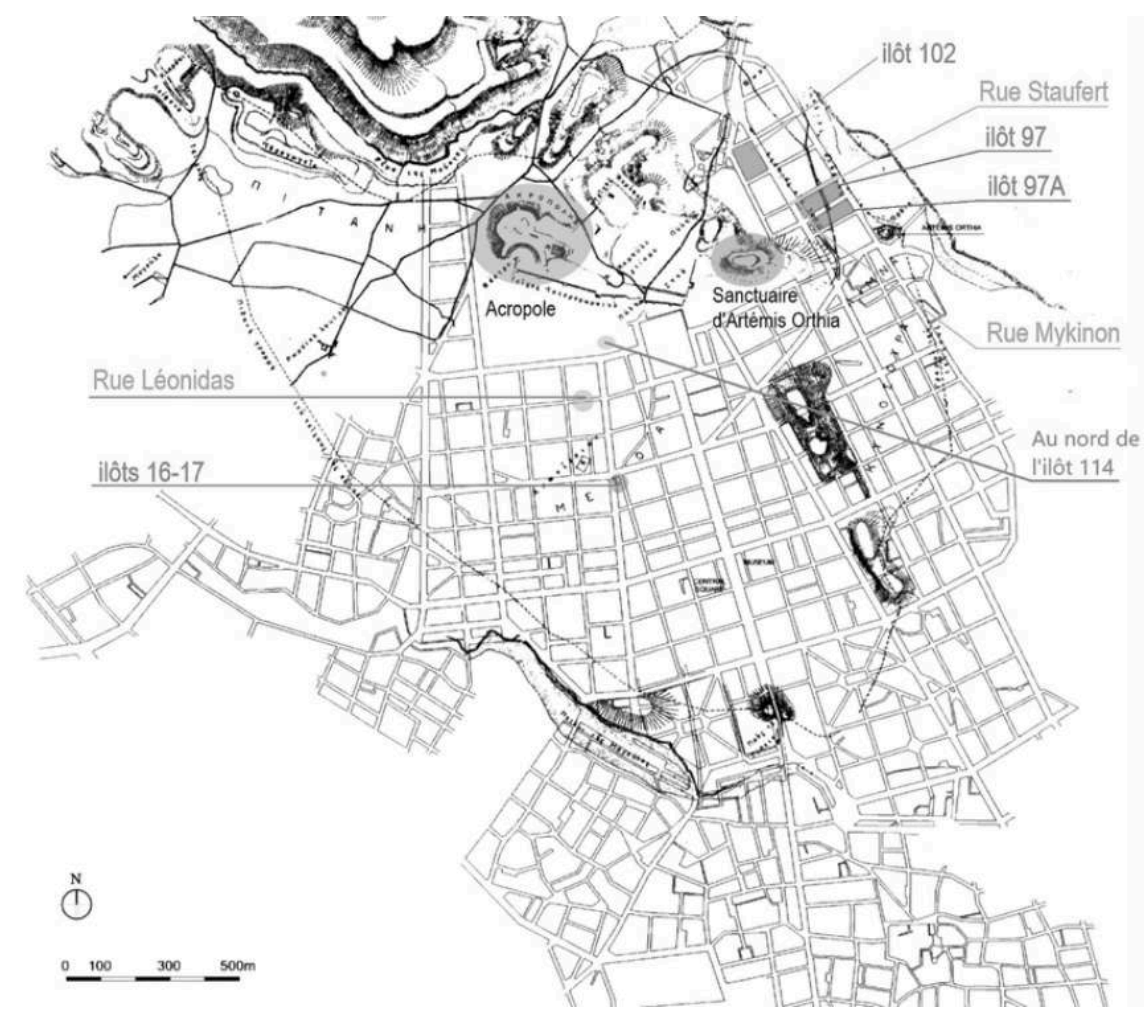


Fig. 4. - Tombe protogéométrique, îlot 97 (d'après Raftopoulou, 1998, fig. 12.14).

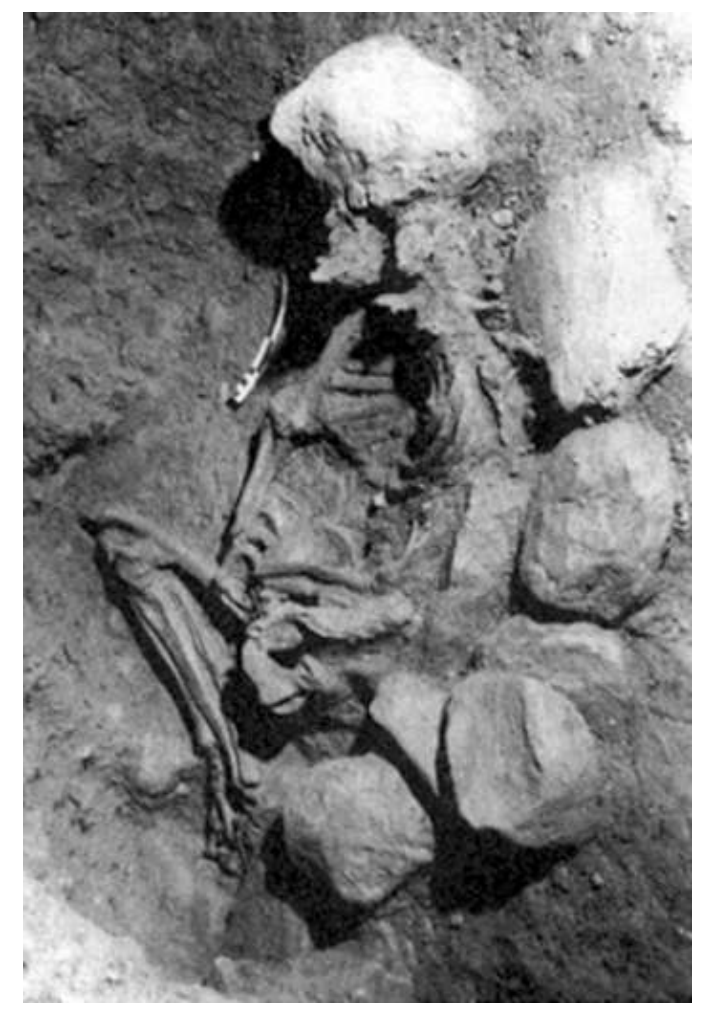

Fig. 5. - Lieu de culte héroïque en rue Staufert sur une tombe de la période géométrique (d'après A. Deltion 51, fig. 11).

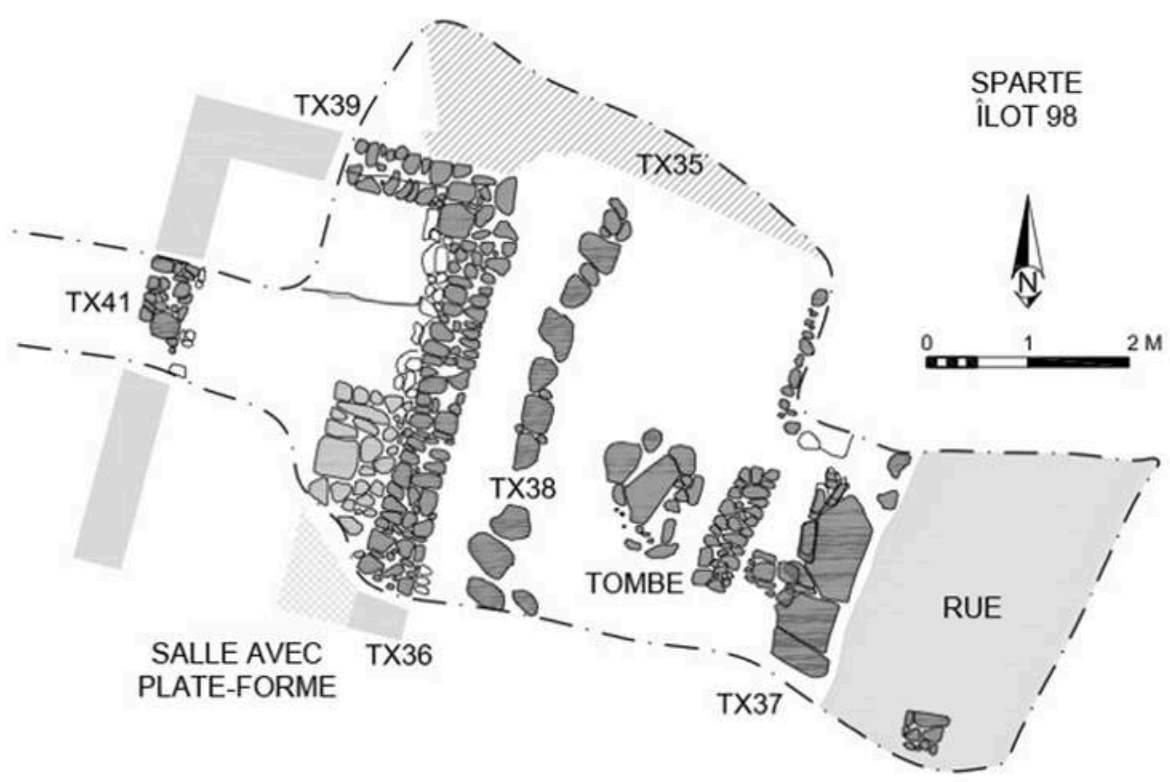

Au sud de l'Acropole, dans la rue Leonidou (entre les îlots 135 et 125), une tombe a été trouvée à l'intérieur d'un grand pithos, contenant les ossements d'une femme et son extraordinaire mobilier funéraire ${ }^{59}$. Dans la zone environnante, un peu plus au nord, juste en-dessous des pentes sud de l'Acropole, il y a d'autres tombes (par exemple au 
nord de l'îlot 114) situées à côté de probables habitations, dont l'existence est une fois encore suggérée par des dépôts céramiques ${ }^{60}$.

D'autres zones semblent participer de la formation de la cité : on pense à la zone comprise entre les îlots 16 et 17, où les fouilleurs ont découvert deux tombes et recueilli des fragments de céramique appartenant probablement à une ou plusieurs maisons ${ }^{61}$. Près de la rive de l'Eurotas, l'Éphorie a identifié une autre tombe avec le défunt en position recroquevillée ${ }^{62}$ et une tombe à l'intérieur d'un pithos ${ }^{63}$. Des traces de peuplement contemporaines aux premiers témoignages de la cité ont été repérées à l'extérieur de la future enceinte de fortification: une nécropole de la période protogéométrique avec 12 tombes été identifiée à Sklavochori ${ }^{64}$.

\section{Les cultes et les institutions dans la création de la polis de Sparte}

À cette époque appartiennent également les premières attestations de culte dans les lieux qui deviendront des sanctuaires urbains et des pôles religieux; ces derniers seront sans doute liés à la configuration progressive d'une communauté politique, qui a par ailleurs défini son identité grâce à la religion : il s'agit de la zone de l'Acropole près du futur temple d'Athéna Poliouchos (fig. 6 : en particulier le mur C-D) ${ }^{65}$, du secteur de Limnai avec le premier autel d'Artémis Orthia de la fin du IX $\mathrm{X}^{\mathrm{e}}$ siècle av. J.-C. (fig. 7, A1) ${ }^{66}$ et d'Amyclées avec le temple d'Apollon ${ }^{67}$. La célébration des fêtes a concouru à la réalisation de l'image de la nouvelle polis et de son unité urbanistique: le rôle du sanctuaire émerge clairement comme un point de repère dans l'étude de la dynamique politico-religieuse qui contribue à la définition de la polis géométrique ${ }^{68}$. 
Fig. 6. - Le sanctuaire d'Athéna Chalkioikos sur l'Acropole avec en évidence la première phase de l'époque Géométrique (d'après Dickins, 1906-1907).

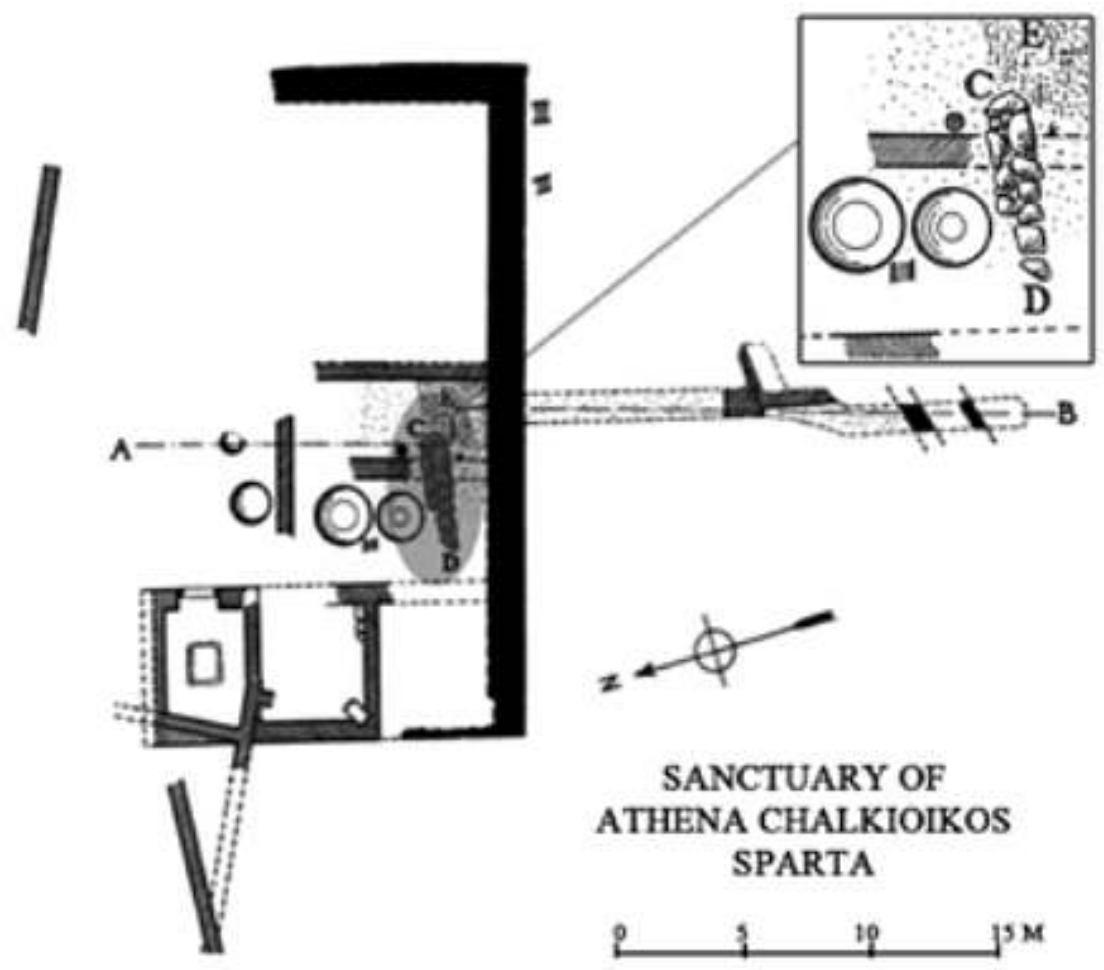

Fig. 7. - Le sanctuaire d'Artémis Orthia dans le Limnai avec le premier autel de la fin IXe siècle av. J.-C. (d'après Dickins, 1906-1907).

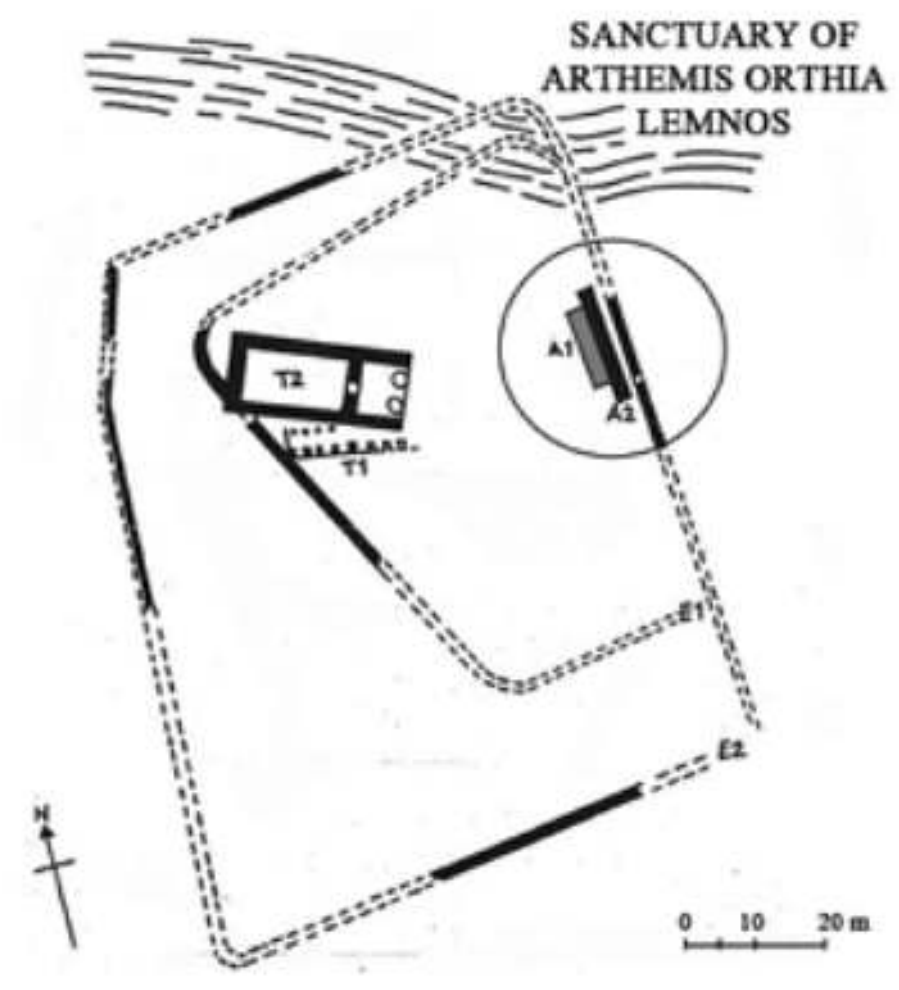


D'autre part, le processus de formation de la polis peut être étudié par la mise en forme des structures politiques et la configuration de l'organisme civique, la communauté de citoyens ${ }^{69}$. Dans les mêmes années où elle consolide ses conquêtes territoriales (guerres de Messénie), la communauté des Spartiates est impliquée dans ce processus d'institutionnalisation et, de fait, elle manifeste un effort précoce dans la mise en place de structures formelles d'organisation de la vie publique, témoignée par un document qui a toujours été considéré exceptionnel, la Grande Rhètra ${ }^{70}$. Le texte nous a été partiellement transmis par Plutarque (Lycurgue, 6), qui l'a présenté comme un oracle reçu par Lycurgue dans le sanctuaire de Delphes. La nature exceptionnelle du document est liée aussi au fait qu'il s'agit de la première constitution d'une polis historiquement attestée. Bien que les datations et interprétations à ce sujet soient nombreuses et contradictoires, il faut sans doute admettre l'existence d'une structure civique qui à Sparte s'est définie au début de son développement, en se configurant comme une entité politique bien précise.

\section{Conclusion}

La nature des traditions et l'état des vestiges disponibles complexifient considérablement la reconstitution des origines de Sparte et la manière dont elle arrive à contrôler non seulement la plaine environnante, mais aussi la partie méridionale de la vallée de l'Eurotas. Nous devons nous contenter d'une interprétation hypothétique incomplète des premières institutions et traditions Spartiates, tenant compte des realia disponibles. Les sources archéologiques prises en considération représentent l'aspect visible des transformations sociales qui ont conduit à la naissance de la polis, les effets et non les causes de ce processus graduel d'agrégation ${ }^{71}$.

La formation de la polis de Sparte s'inscrit dans un contexte de crise et de changements importants, qui concernent non seulement la Laconie mais plus généralement la Grèce tout entière pendant les Dark Ages. Après la crise et la chute des palais mycéniens, le vide administratif et même démographique est comblé par un peuple venant probablement du nord, qui se mélange progressivement aux populations autochtones. Dans ce sens, on peut comprendre la discontinuité d'occupation spatiale ainsi que la discontinuité linguistique. Le nouveau peuple décide de s'installer dans une zone qui était auparavant presque inhabitée et considérée comme étant périphérique par rapport aux centres de l'âge du bronze tardif. Des zones d'habitations se créent progressivement autour de la colline de l'Acropole. Depuis les périodes protogéométrique et géométrique, Sparte sacralise quelques endroits et les valorise en tant que points de repère de la communauté; elle jette également les bases d'une organisation de l'espace urbain kata kômas, structure qui ne diffère pas de celle de la majorité des poleis de cette période. La cohésion territoriale de ces rassemblements dispersés est assurée par les cultes civiques qui maintiennent le caractère commun de la nouvelle réalité politique. La polis kata kômas visitée et décrite par Thucydide est née, avec sa propre constitution et ses cultes, mais reste fragmentée au niveau territorial. 


\section{BIBLIOGRAPHIE}

\section{Abréviation}

- IG = Inscriptiones Graeciae, Berlin, G. de Gruyter, 1873-.

ARAVANTINOS Vasilis, GODART Louis \& SACCONI Anna, Thèbes. Fouilles de la Cadmée, vol. I : Les tablettes en linéaire B de la odos Pelopidou. Édition et commentaire, Pise, Istituti editoriali e poligrafici internazionali, 2001.

ARAVANTINOS Vasilis, GODART Louis \& SACCONI Anna, Thèbes. Fouilles de la Cadmée, vol. III : Corpus des documents d'archives en linéaire B de Thèbes (1-433), Pise, Istituti editoriali e poligrafici internazionali, 2002.

ARAVANTINOS Vasilis \& VASILOGAMVROU Adamantia, « The First Linear B Documents from Ayios Vasileios (Laconia) », dans P. Carlier (éd.), Études mycéniennes 2010 (Actes du XIII ${ }^{\mathrm{e}}$ colloque international sur les textes égéens, Sèvres, Paris, Nanterre, 20-23 septembre), Pise, Serra, 2012, p. 41-54.

BANOU Emilia, Beitrag zum Studium Lakoniens in der mykenischen Zeit, Munich, Tuduv, 1996.

BANOU Emilia, « New Evidence in Early Helladic Laconia », BSA, 94, 1999, p. 63-79.

BANOU Emilia, « Middle Helladic Laconia: New Evidence », SMEA, 42, 2000, p. 175-199.

BANOU Emilia, «The Mycenaean Presence in the Southeastern Eurotas Valley: Vouno Panagias and Agios Georgios ", dans W. G. Cavanagh, C. Gallou \& M. Georgiadis (éd.), Sparta and Laconia: From Prehistory to Pre-Modern (Proceedings of the Conference held in Sparta, 17-20 March 2005), BSA, 16, 2009, p. 77-84.

CALLIGAS Paul, « From the Amyklaion », dans J. M. Sanders (éd.), Philolakon: Lakonian Studies in Honour of Hector Catling, Londres, The British School at Athens, 1992, p. 31-48.

CARTLEDGE Paul, « Early Lacedaimon: The Making of a Conquest-State », dans J. M. Sanders (éd.), Philolakon: Lakonian Studies in Honour of Hector Catling, Londres, The British School at Athens, 1992, p. 49-55.

CARTLEDGE Paul, « City and Chora in Sparta: Archaic to Hellenistic », dans W. G. Cavanagh \& S. E. C. Walker (éd.), Sparta in Laconia (Proceedings of the 19th British Museum Classical Colloquium, London, 6-8 December 1995), BSA, 4, Londres, The British School at Athens, 1998, p. 39-47.

CATLING Hector William, « Sparta. Excavation at the Menelaion », Archaiologikon Deltion, 30 B'1, 1975, p. 80-85.

CATLING Hector William, « New Excavations at the Menelaion, Sparta », dans U. Jantzen (éd.), Neue Forschungen in griechischen Heiligtümern (Internationales Symposium in Olympia vom 10.-12. Oktober 1974 anlässlich der Hundertjahrfeier der Abteilung Athen und der deutschen Ausgrabungen in Olympia), Tubingue, Wasmuth, 1976.

CATLING Hector William, « Excavations at the Menelaion, Sparta 1973-76 », AR, 23, 1977a, p. 24-42.

CATLING Hector William, « Excavations at the Menelaion, Sparta 1976-77 », Lakonikai Spoudai, 3, 1977b, p. 408-416. 
CATLING Hector William, « Excavations and Study at the Menelaion, Sparta, 1978-81 », Lakonikai Spoudai, 4, 1982, p. 28-43.

CATLING Hector William, « Study at the Menelaion, 1982-83 », Lakonikai Spoudai, 7, 1983, p. 23-31.

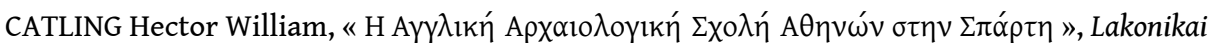
Spoudai, 8, 1986, p. 189-204.

CATLING Hector William, " The Work of the British School at Athens at Sparta and in Laconia », dans W. G. Cavanagh \& S. E. C. Walker (éd.), Sparta in Laconia (Proceedings of the 19th British Museum Classical Colloquium, London, 6-8 December 1995), BSA, 4, Londres, The British School at Athens, 1998, p. 19-27.

CATLING Hector William, Sparta: Menelaion I. The Bronze Age, BSA, Suppl. 45, Londres, The British School at Athens, 2009.

CAVANAGH William G. \& CROUWEL Joost H., « Melathria: A Small Mycenaean Rural Settlement in Laconia », dans J. M. Sanders (éd.), Philolakon: Lakonian Studies in Honour of Hector Catling, Londres, The British School at Athens, 1992, p. 77-86.

CAVANAGH William G., CROUWEL Joost H. \& CATLING Richard W. V., Continuity and Change in a Greek Rural Landscape: The Laconia Survey, vol. I : Methodology and Interpretation, BSA, Suppl. 26, Londres, The British School at Athens, 1996.

CAVANAGH William G., CROUWEL Joost H. \& CATLING Richard W. V., Continuity and Change in a Greek Rural Landscape: The Laconia Survey, vol. II : Archaeological Data, BSA, Suppl. 27, Londres, The British School at Athens, 1996.

CAVANAGH William G., MEE Christopher Brian \& JAMES Peter, The Laconia Rural Sites Project, BSA, Suppl. 26, Londres, The British School at Athens, 2005.

CHAPIN Anne P., DAVIS Brent, HITCHCOCK Louise A. \& BANOU Eleni, « The Vaphio Tholos Tomb and the Construction of a Symbolic Landscape in Laconia, Greece », dans G. Touchais, R. Laffineur \& F. Rougemont (éd.), Physis : l'environnement naturel et la relation homme-milieu dans le monde égéen protohistorique (Actes de la $14^{\mathrm{e}}$ Rencontre égéenne internationale, Paris, Institut national d'histoire de l'art (INHA), 11-14 décembre 2012), Aegaeum, 37, Louvain, Peeters, 2014, p. 145-152. COOK John Manuel \& NICHOLLS Richard, « Laconia », BSA, 45, 1950, p. 161-298.

COULSON William D. E., « The Dark Age Pottery of Sparta », BSA, 80, 1985, p. 29-84.

COULSON William D. E., The Dark Age Pottery of Messenia, Göteborg, Paul Åström, coll. « Studies in Mediterranean Archaeology and Literature, 43 », 1986.

COULSON William D. E., « The Dark Age Pottery of Sparta, 2. Vrondama », BSA, 83, 1988, p. 21-24. DAWKINS Richard McGillivray et al., The Sanctuary of Artemis Orthia at Sparta, Londres, Macmillan, 1929.

DEMAKOPOULOU Katie, « A Mycenaean Pictorial Vase of the Fifteenth Century B.C. from Laconia », BSA, 66, 1971, p. 95-100.

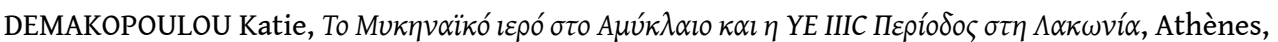
1982.

DEMAKOPOULOU Katie, « The Early Cult at the Amyklaion the Mycenean Sanctuary », dans A. Delivorrias \& S. Vlizos (éd.), Amykles Research Project: Works 2005-2010 (Benaki Museum 11-12, 2011-2012), 2015, p. 105-112. 
DICKINS Guy, « Laconia. Excavations at Sparta, 1907. The Hieron of Athena Chalkioikos », BSA, 13, 1906-1907, p. 137-154.

DICKINS Guy, « Laconia. Excavations at Sparta, 1908. The Hieron of Athena Chalkioikos ", BSA, 14, 1907-1908, p. 142-146.

DICKINSON Oliver Thomas Pilkington Kirwan, « Reflections Bronze Age Laconia », dans J. M. Sanders (éd.), Philolakon: Lakonian Studies in Honour of Hector Catling, Londres, The British School at Athens, 1992, p. 109-114.

EDER Birgitta, Argolis, Lakonien, Messenien. Vom Ende der mykenischen Palastzeit bis zur Einwanderung der Dorier, Vienne, Verlag der Österreichischen Akademie der Wissenschaften, 1998.

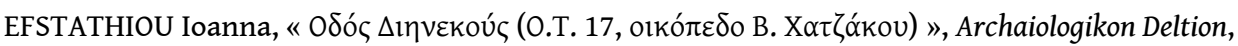
52 B'1, 1997, p. 190.

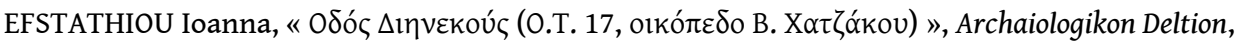
54 B'1, 1999, p. 172-174.

FINLEY Moses I., « Sparta », dans J.-P. Vernant (éd.), Problèmes de la guerre en Grèce ancienne, Paris/ La Haye, Mouton \& Co, 1968, p. 143-160.

FLOURIS Christos G., « ANA Archaiologikon Deltion, 51 B'1, 1996, p. 123-125.

GEORGOULAKI Eleni, « Le type iconographique de la statue cultuelle d'Apollon Amyklaios : un emprunt oriental ? », Kernos, 7, 1994, p. 95-118.

GRECO Emanuele, « Dal villaggio alla città », dans J. Guilaine \& S. Settis (éd.), Storia d'Europa. Preistoria e Antichità, vol. 2, Turin, G. Einaudi, 1994, p. 587-600.

GRECO Emanuele, « With Pausanias (and Others) in the Agora of Sparta », dans A. Ercolani \& M. Giordano (éd.), Submerged Literature in Ancient Greek Culture, vol. 3 : The Comparative Perspectives, Berlin/Boston, De Gruyter, 2016, p. 113-130.

HODKINSON Stephen, Property and Wealth in Classical Sparta, Swansea, The Classical Press of Wales, 2000.

HODKINSON Stephen, « Sparta and Nazi Germany in Mid-20th-century British Liberal and LeftWing Thought ", dans A. Powell \& S. Hodkinson (éd.), Sparta: The Body Politic, Swansea, Classical Press of Wales, 2010, p. 297-342.

HODKINSON Stephen \& MORRIS Ian MacGregor (éd.), Sparta in Modern Thought. Politics, History and Culture, Oxford, Classical Press of Wales, 2012.

HOPE SIMPSON Richard, « The Mycenaean Settlements in the Sparta Plain and the Ancient Traditions », SMEA, 51, 2009, p. 315-335.

HOPE SIMPSON Richard \& DICKINSON Oliver Thomas Pilkington Kirwan, A Gazetteer of Aegean Civilization in The Bronze Age, vol. I : The Mainland and Islands, Göteborg, Paul Åström, coll. « Studies in Mediterranean Archaeology, 52 », 1979.

KÕIV Mait, Ancient Tradition and Early Greek History: The Origins of States in Early-Archaic Sparta, Argos and Corinth, Tallinn, Avita, 2003.

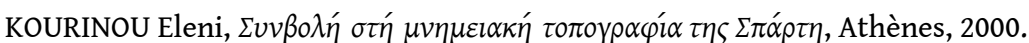

KOUROU Nota, « The Settlements of the Dark Ages », dans A. P. Lagopoulos (éd.), A History of the Greek City, Oxford, Archaeopress, 2009, p. 109-123. 


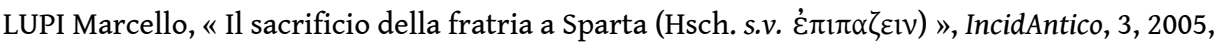
p. 199-214.

LUPI Marcello, « Amompharetos, the lochos of Pitane and the Spartan system of villages », dans S. Hodkinson \& A. Powell (éd.), Sparta and War, Swansea, Classical Press of Wales, 2006, p. 185-218.

LUPI Marcello, « Le origini di Sparta e il Peloponneso arcaico », dans M. Giangiulio (éd.), Storia d'Europa e del Mediterraneo. Il mondo antico. II : La Grecia, Rome, Salerno Editrice, 2008, p. 363-393.

LUPI Marcello, « Testo e contesti. La Grande Rhetra e le procedure spartane di ammissione alla cittadinanza », IncidAntico, 12, 2014, p. 9-41.

LUPI Marcello, Sparta. Storia e rappresentazioni di una città greca, Rome, Carocci, 2017.

LUPI Marcello, «Citizenship and Civic Subdivisions: The Case of Sparta », dans A. Duplouy \& R. Brock (éd.), Defining Citizenship in Archaic Greece, Oxford, Oxford University Press, 2018, p. $161-178$.

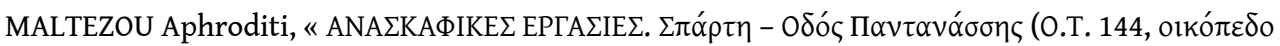
П. Вıбßи́)», Archaiologikon Deltion, 67 (2012), Chroniques, 2016, p. 98-99

MARCHAND Suzanne L., Down from Olympus. Archaeology and Philhellenism in Germany, 1750-1970, Princeton, Princeton University Press, 1996.

MORGAN Catherine, The Late Bronze Age Settlement and the Early Iron Age Sanctuary, Princeton, American School of Classical Studies at Athens, 1999.

MUSTI Domenico, « Linee di sviluppo istituzionale e territoriale tra miceneo e alto arcaismo », dans D. Musti, A. Sacconi, L. Rocchetti et al. (éd.), La transizione dal Miceneo all'alto Arcaismo. Dal palazzo alla città (Atti del convegno internazionale, Roma, 14-19 marzo 1988), Rome, Consiglio Nazionale delle Ricerche, 1991, p. 15-33.

NAFISSI Massimo, «Sparta », dans K. A. Raaflaub \& H. van Wees (éd.), A Companion to Archaic Greece, Chichester/Malden (MA), Blackwell Publishing, 2009, p. 117-137.

NAFISSI Massimo, « Esparta », dans C. Fornis (éd.), Mito y arqueología en el nacimiento de ciudades legendarias de la Antigüedad, Séville, Universidad de Sevilla, 2012, p. 25-57.

NILSSON Martin P., The Minoan-Mycenaean Religion and Its Survival in Greek Religion, Lund, Gleerup, 1950 (2e éd.).

OLLIER François, Le Mirage Spartiate. Étude sur l'idéalisation de Sparte dans l'antiquité grecque de l'origine jusqu'aux Cyniques, Paris, De Boccard, 1933.

PARETI Luigi, « Le tribù personali e le tribù locali a Sparta », RAL, 19, 1910, p. 455-73.

PARETI Luigi, Storia di Sparta arcaica, Florence, F. LeMonnier, 1917.

PETROPOULOU Angeliki, « Hyakinthos and Apollo of Amyklai: Identities and Cults. A Reconsideration of the Written Evidence ", dans A. Delivorrias \& S. Vlizos (éd.), Amykles Research Project: Works 2005-2010 (Benaki Museum 11-12, 2011-2012), 2015, p. 153-161.

PETTERSSON Michael, Cults of Apollo at Sparta. The Hyakinthia, the Gymnopaidiai and the Karneia, Stockholm, Paul Åström, 1992.

POLIGNAC François de, « Repenser la "cité" ? Rituels et société en Grèce archaïque », dans M. H. Hansen \& K. Raaflaub (éd.), Studies in the Ancient Greek Polis, Stuttgart, F. Steiner, coll. « Historia Einzelschriften, 95 », 1995, p. 7-19. 
POLIGNAC François de, « Mediation, Competition, and Sovereignty: The Evolution of Rural Sanctuaries in Geometric Greece », dans S. E. Alcock \& R. Osborne (éd.), Placing the Gods: Sanctuaries and Sacred Space in Ancient Greece, Oxford, Clarendon Press, 1996, p. 3-18.

POLIGNAC François de, «Forms and Processes: Some Thoughts on the Meaning of Urbanization in Early Archaic Greece ", dans R. Osborne \& B. Cunliffe (éd.), Mediterranean Urbanization 800-600 BC, Oxford/New York, Oxford University Press, 2005, p. 45-69.

PROIETTI Giorgia, « Memoria collettiva e identità etnica. Nuovi paradigmi teorico-metodologici nella ricerca storica », dans E. Franchi \& G. Proietti (éd.), Forme della memoria e dinamiche identitarie nell'antichità greco-romana, Trente, Università degli Studi di Trento, 2012, p. 13-41.

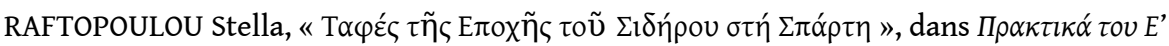

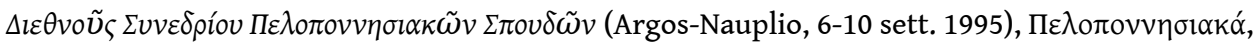

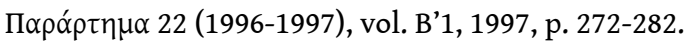

RAFTOPOULOU Stella, « New Finds from Sparta », dans W. G. Cavanagh \& S. E. C. Walker (éd.), Sparta in Laconia (Proceedings of the 19th British Museum Classical Colloquium, London, 68 December 1995), BSA, 4, Londres, British School at Athens, 1998, p. 125-140.

RAFTOPOULOU Stella, « Contributo alla topografia di Sparta durante l'età geometrica ed arcaica », dans Taranto e il Mediterraneo (Atti del XLI Convegno di studi sulla Magna Grecia, Taranto, 12-16 ottobre 2001), Tarente, Istituto per la storia e l'archeologia della Magna Grecia, 2001, p. 25-42.

ROCHE Helen, Sparta's German Children, Oxford/Cambridge, The Classical Press of Wales, 2013.

ROUSSEL Denis, Thucydide. La guerre du Péloponnèse, édition et traduction du grec ancien par D. Roussel, préface de Pierre Vidal-Naquet, Paris, Gallimard, 2000.

SAKKA Nike, "The Excavation of the Ancient Agora of Athens: The Politics of Commissioning and Managing the Project », dans D. Damaskos \& D. Plantzos (éd.), A Singular Antiquity. Archaeology and Hellenic Identity in Twentieth-Century Greece, Athènes, Benaki Museum (3rd Suppl.), 2008,

p. 111-124.

SCHNAPP-GOURBEILLON Annie, Aux origines de la Grèce (XIII'-VIII siècles avant notre ère) : la genèse du politique, Paris, Les Belles Lettres, 2002.

SIRANO Francesco, « Fuori da Sparta. Note di topografia lacone. Recenti studi e nuovi dati dal territorio », ASAtene, 74-75 (1996-1997), 2000, p. 397-465.

SMITH David Michael, « Archaeology in Greece 2016-2017. Recent Research in Early Helladic Southern Greece », Archaeological Reports, 63, 2016-2017, p. 107-29.

SPALLINO Giovanni, «Athena Chalkioikos a Sparta: riesame dei dati archeologici e topografici del santuario ", dans F. Longo, R. Di Cesare \& S. Privitera (éd.), Dromoi. Studi sul mondo antico offerti a Emanuele Greco dagli allievi della Scuola Archeologica Italiana di Atene, Paestum, Pandemos, 2016, p. 571-586.

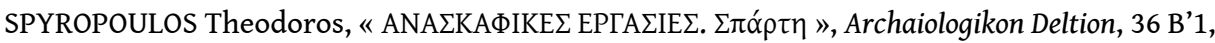
1981a, p. 121-125.

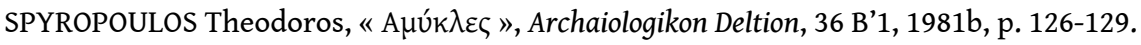

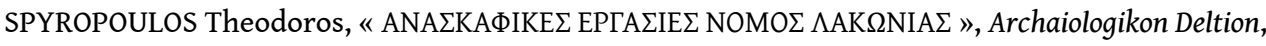
37 B'1, 1982, p. 111-121.

SPYROPOULOS Theodoros, "Pellana, the Administrative Centre of Prehistoric Laconia », dans W. G. Cavanagh \& S. E. C. Walker (éd.), Sparta in Laconia (Proceedings of the 19th British Museum 
Classical Colloquium, London, 6-8 December 1995), BSA, 4, Londres, The British School at Athens, 1998, p. 28-38.

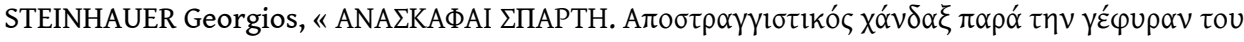

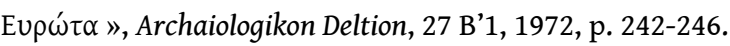

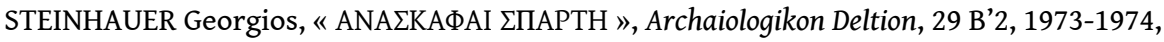
p. 283-292

STEWART Daniel R., « "Most Worth Remembering”: Pausanias, Analogy, and Classical Archaeology », Hesperia, 82, 2013, p. 231-261.

THEMOS Athanassios \& ZAVVOU Eleni, « Recent Finds of Child Burials in the Area of Ancient Sparta from Protogeometric to Roman Times », dans A. M. Guimier-Sorbets (éd.), L'enfant et la mort dans l'Antiquité, vol. I : Nouvelles recherches dans les nécropoles grecques. Le signalement des tombes d'enfants, Paris, De Boccard, 2010, p. 227-241.

TIGERSTEDT Eugène Napoleon, The Legend of Sparta in Classical Antiquity, Stockholm, Alqmvist \& Wiksell, coll. «Stockholm Studies in History of Literature, 9, 15 », 1965-1974.

TOSTI Valeria, « Una riflessione sui culti eroici nella Sparta katà komas. L'edificio con banchina di odos Staufert », ASAA, 89, 2011, p. 95-108.

TOSTI Valeria, « La nascita di Sparta: per una revisione critica degli indicatori archeologici », dans M. Scafuro \& A. Pontrandolfo (éd.), Dialoghi sull'Archeologia della Magna Grecia e del Mediterraneo (Atti del I Convegno internazionale di studi, Paestum, 7-9 settembre 2016), Paestum, Pandemos, 2017, p. 193-206.

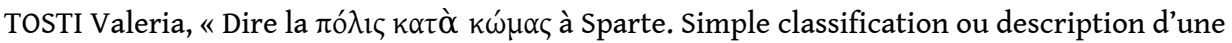
réalité urbaine ? ", dans L. Lopez-Rabetel, V. Mathé \& J. C. Moretti (éd.), Dire la ville en grec aux époques antique et byzantine (Actes du colloque de Créteil, Paris, 10-11 juin 2016), Lyon, MOM éditions, 2020, p. 172-192.

TSOULI Maria, TSIAGGOURIS Giorgos \& PAPADOPOULOS Vasilios, « ANA

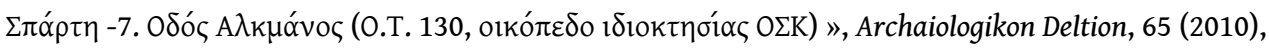
Chroniques, p. 440-443.

WACE Alan John Bayard, THOMPSON Maurice Scott \& DROOP John Percival, « Laconia I. Excavations at Sparta, 1909. The Menelaion », BSA, 15, 1909-09, p. 108-157.

TSOUNTAS Christos, « Anaskaphai taphōn en Mykēnais », Archaiologike Hetaireia, 6, 1888, p. $119-180$.

TSOUNTAS Christos, «Ereunai en tē Lakōnikē kai o taphos tou Bapheiou », Archaiologike Hetaireia, 7, 1889, p. 129-172.

TSOUNTAS Christos \& MANATT James Irving, The Mycenaean Age: A Study of the Monuments and Culture of Pre-Homeric Greece, Londres, MacMillan, 1897.

VERMEULE Emily, Greece in the Bronze Age, Chicago, University of Chicago Press, 1964.

VLACHOPOULOS Andreas, Archaiologia: Peloponnesos, Athènes, Ekdotikós Oíkos Mélissa, 2012.

VLACHOU Vicky, «The Spartan Amyklaion: The Early Iron Age Pottery from the Sanctuary »,

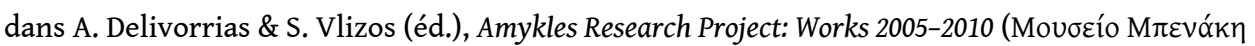
11-12, 2011-2012), 2015, p. 113-124.

VLACHOU Vicky, « From Mycenean Cult Practice to the Hyakinthia Festival of the Spartan Polis. Cult Images, Textiles and Ritual Activity at Amykles: An Archaeological Perspective », dans 
A. Tsingarida \& I. S. Lemos (éd.), Constructing Social Identities in Early Iron Age and Archaic Greece, Bruxelles, CreA-Patrimoine, 2017, p. 11-42.

VLIZOS Stavros, «The Amyklaion Revisited: New Observations on a Laconian Sanctuary of Apollo », dans N. Kaltsas (éd.), Athens-Sparta. Contribution to the Research on the History and Archaeology of the Two City-States, New York/Athènes, Onassis Foundation, 2009, p. 11-23.

VLIZOS Stavros, « Amykles Research Project: Excavation Works 2005-2010 », dans A. Delivorrias

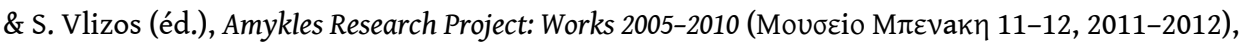
p. 91-104.

WADE-GERY Henry Theodore, « The Growth of the Dorian States », CAH, III, 1925, p. 527-70.

WADE-GERY Henry Theodore, « The Spartan Rhetra in Plutarch, Lycurgus VI: C. What Is the Rhetra? », CQ, 38 (3/4), 1944, p. 115-126.

WATERHOUSE Helen, The British School at Athens: The First Hundred Years, BSA, Suppl. 19, Londres, Thames and Hudson, 1986.

WATERHOUSE Helen \& HOPE SIMPSON Richard, « Prehistoric Laconia: Part I », BSA, 55, 1960, p. 67-101.

WATERHOUSE Helen \& HOPE SIMPSON Richard, « Prehistoric Laconia: Part II », BSA, 56, 1961, p. 114-161.

WEES Hans van, « Tyrtaeus' Eunomia: Nothing to Do with the Great Rhetra », dans S. Hodkinson \& A. Powell (éd.), Sparta: New Perspectives, Swansea, The Classical Press of Wales, 1999, p. 1-42.

WOODWARD Arthur Maurice, « Laconia. Excavations at Sparta, 1924-25. The Acropolis. The Finds », BSA, 26, 1923-1925, p. 253-276.

WOODWARD Arthur Maurice, « Laconia. Excavations at Sparta, 1927. The Acropolis », BSA, 28, 1926-1927, p. 37-48.

WOODWARD Arthur Maurice \& HOBLING Margaret Blanche, « Laconia. Excavations at Sparta, 1924-25. The Acropolis ", BSA, 26, 1923-1925, p. 240-252.

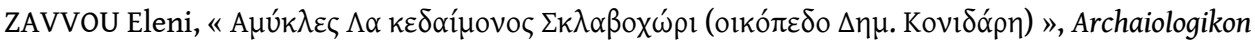
Deltion, 51 B'1, 1996, p. 129-131.

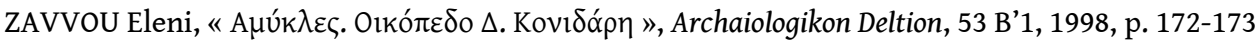

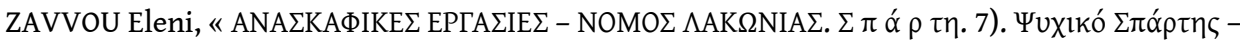

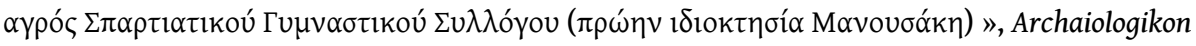
Deltion, 54 B’1, 1999, p. 165-166.

ZAVVOU Eleni, «Archaeological Finds from the Area of Anthochori », dans W. G. Cavanagh, C. Gallou \& M. Georgiadis (éd.), Sparta and Laconia: from Prehistory to Pre-modern Times (Proceedings of the Conference held in Sparta, 17-20 March 2005), BSA, 16, Londres, The British School at Athens, 2009, p. 29-42.

ZAVVOU Eleni \& THEMOS Athanassios, « Sparta from Prehistoric to Early Christian Times: Observations from the Excavations of 1994-2005 », dans W. G. Cavanagh, C. Gallou \& M. Georgiadis (éd.), Sparta and Laconia: From Prehistory to Pre-Modern Times (Proceedings of the Conference held in Sparta, 17-20 March 2005), BSA, 16, Londres, British School at Athens, 2009, p. 105-122.

ZURBACH Julien, « La formation des cités grecques. Statuts, classes et systèmes fonciers ", Annales, 68, 2013/2014, p. 957-998. 


\section{NOTES}

1. Thucydide, I, 10, 2: «Ainsi, à supposer que Sparte soit dévastée et que seuls subsistent les sanctuaires et les fondations de ses édifices [...]. Néanmoins, parce que les sanctuaires et les monuments sont modestes, parce que, comme c'était autrefois le cas dans toute la Grèce, les Lacédémoniens vivent disséminés en bourgades, on pourrait croire que Sparte n'était qu'une puissance de second ordre. » Trad. Roussel (2000).

2. Stewart $(2013,139)$.

3. L'enquête a été réalisée en particulier par Hodkinson (2010), Hodkinson \& Mc Morris (2012) et par Sakka (2008) spécifiquement pour la reconstruction du climat politique américain et grec précédent le début de fouilles dans l'agora du Kerameikos à Athènes. Voir aussi Marchand (1996) à propos du philhellénisme allemand dans les années 1933-1945, Roche (2013) et Greco (2016, 115-118).

4. À propos de l'activité de la British School at Athens à Sparte, voir Catling $(1986,189-204)$, Waterhouse (1986, 105-107), Catling (1998, 17-29) et Cartledge (1998, 46-47). Pour les fouilles dans le sanctuaire d'Artémis Orthia, voir Dawkins et al. (1929). Pour les fouilles sur l'acropole, voir Dickins (1906-1907, 137-154), Dickins (1907-1908, 142-146), Woodward-Hobling (1923-1925, 240-252, 253-276), Woodward-Hobling (1926-1927, 37-48).

5. Kourinou $(2000,16)$.

6. Ollier (1938) ; Tigersedt (1965) : « légende de Sparte».

7. Sur l'invention de la tradition et la sociologie de la mémoire, voir Proietti (2012), avec bibliographie précédente.

8. Barthélemy, puis Leake en 1805-1806.

9. Pareti (1910) et Pareti (1917, 173-176); Wade-Gery (1925, 560-561) et Wade-Gery (1944, 117-123). Pour un approfondissement sur la naissance et le développement de ce modèle des cinq villages de Sparte, voir Lupi (2006, 2007 et 2014).

10. Pausanias, dans le livre III, 16, 9, parle de l'étiologie du rite de diamastigosis dans le sanctuaire d'Artémis Orthia. Voir aussi l'inscription IG V 1, 1674-1688; différentes inscriptions font référence aux sphaireis et à leurs courses du $\mathrm{I}^{\mathrm{er}}-\mathrm{II}^{\mathrm{e}}$ siècle, ou encore du début du III ${ }^{\mathrm{e}}$ siècle apr. J.-C. Voir Tosti (2020).

11. Lupi (2005) et Lupi $(2006,204-207)$; Lupi (2008) sur la centralité de Pitanè suggérée par les sources antiques. Voir aussi Nafissi (2012, 41-44).

12. Pausanias, dans le livre III, 16,9 en parlant de l'étiologie du rite de diamastigosis dans le sanctuaire d'Artémis Orthia, cite les kômai spartiates. Voir aussi l'inscription IG V 1, 1674-1688 ; différentes inscriptions $\mathrm{du} \mathrm{I}^{\mathrm{er}}-\mathrm{II}^{\mathrm{e}}$ siècle, ou encore du début du $\mathrm{III}^{\mathrm{e}}$ siècle apr. J-C. font référence aux sphaireis et à leurs courses, et aux kômai de Sparte.

13. Homère, Iliade, II, 581-582.

14. Waterhouse \& Hope Simpson (1960 et 1961) ; Hope Simpson \& Dickinson (1979, 107-125); Demakopoulou (1982) ; Cavanagh, Crouwel \& Catling (1996 et 2002) ; Cavanagh, Mee \& James (2005) ; Banou (1996, 1999, 2000 et 2009).

15. Homère, Iliade, II, 581-590.

16. Les tablettes ont été trouvées dans la rue Pelopidou à Thèbes : voir Aravantinos, Godart \& Sacconi (2001 et 2002).

17. Lupi $(2017,43)$. Le terme mycénien «Lacédémon » révèle l'importance attribuée à la phase achéenne, revendiquant une certaine continuité historique.

18. Homère, Odyssée, XIII, 414-415 ; XV, 1-4 ; XI, 460 ; Pindare, Olympiques, 6, 28-31 ; Terpandre dans Plutarque, Lycurgue, 21, 4-5.

19. Woodward (1926-1927, 38-39); Cook \& Nicholls (1950, 298, fig. 19); Waterhouse \& Hope Simpson (1960, 70) ; Dawkins et al. (1929, 378-379, 380, fig. 144 e-f) ; Zavvou-Themos (2009); Tsouli, Tsiaggouris \& Papadopoulos (2010, 442) ; Maltezou (2012, 98-99) ; Smith (2016-2017, 114). 
20. Au centre de la ville moderne, à l'est de l'avenue Konstantinou Palaiologou.

21. Zavvou (1999, 165-166); Zavvou \& Themos (2009, 110-111) ; Maltezou (2012, 98-99).

22. Zavvou \& Themos (2009); Maltezou $(2012,93)$.

23. Voir Catling (2009) avec bibliographie précédente.

24. Mansion 1 : Megaron - pièces 6-9, deux couloirs sur les côtés - salles 5 et 10 - qui donnaient accès à une série de pièces mineures pour le stockage - pièces 1-4 à l'ouest ; pièces 11-15 à l'est.

25. Mansion 2 : bâtiment à deux étages.

26. Mansion 3 : détruit par un incendie.

27. Wace, Thompson \& Droop (1908-1909); Catling (1976, 1977a, 1977b, 1982 et 1983); Catling (1975, 80-85) ; Catling (2009).

28. Tsountas en 1890 ; Furtwängler en 1904 ; Fiechter en 1907 ; Buschor en 1925 ; DelivorriasVlizos de 2005 à aujourd'hui.

29. Vlizos (2009, 1-17) et Vlizos (2015) ; Demakopoulou (2015), avec bibliographie précédente.

30. Calligas (1992, 39-40). Voir Georgoulaki (1994, 95-96) et Sirano (1995) qui datent les rares attestations submycéniennes au plus tard de 1050 av. J.-C.

31. Nilsson (1950, 470 et 556), Demakopoulou (1982, 82-96, 174-175). Voir aussi Dickinson (1992), Morgan (1999, 382-384, 390).

32. Spyropoulos (1981b, 126-129, fig. $60 \alpha-\delta$, pl. 2-5) ; Zavvou (1998, 172-173).

33. Spyropoulos (1982, 111-121); voir aussi Spyropoulos (1998).

34. Demakopoulou (1971, 95-100); Cavanagh \& Crouwel (1992).

35. Tsountas $(1889,129-172)$; Tsountas \& Manatt $(1897,130,144-45)$; Vermeule $(1964,127-130)$.

36. Spyropoulos $(1982,112$, fig. $60 \alpha-\beta)$.

37. Tsountas $(1888,198)$; Chapin, Davis, Hitchcock \& Banou $(2014,146)$.

38. Waterhouse \& Hope Simpson $(1960,80-81)$; Hope Simpson \& Dickinson $(1979,110)$; Hope Simpson (2009, 322-23) ; Banou (1996) et Banou (1999, 65).

39. Les tablettes publiées ont été trouvées hors contexte et datent probablement entre le XIV et le XII ${ }^{\mathrm{e}}$ siècle. Voir Aravantinos \& Vasilogamvrou (2012, 41-54).

40. Ergon [To Ergon tis archaiologikis eterias] (2010, 33-40 ; 2011, 29-31; 2012, 50-53 ; 2013, 27-29 ; 2014, 22-24; 2016, 19-20).

41. Il s'agit pour Musti (1991) d'une crise principalement politico-institutionnelle puis économique. Voir aussi Schnapp-Gourbeillon (2002). Plus récemment, on voudrait redimensionner le concept de siècles obscurs : cf. Kourou (2009). À propos des effets de la chute des palais mycéniennes, voir Zurbach (2013/2014, 994-995).

42. Sur la base des prospections menées en Laconie, au HR IIIB correspondent 50 sites, nombre décroissant à 15 unités au HR IIIC. Parmi ces sites, seulement 4 survivent au $\mathrm{XI}^{\mathrm{e}}$ siècle av. J.-C., dont Amyclées est le seul dans la moyenne vallée de l'Eurotas. Cf. Cartledge (1992, 51).

43. L'étude a été réalisée sur des bases purement stylistiques: Vlachou (2015, 113-114, avec bibliographie précédente aux notes $8-11$; 2017) veut soutenir la continuité de l'utilisation de l'espace sur la colline entre la fin du $\mathrm{XI}^{\mathrm{e}}$ et le début du $\mathrm{X}^{\mathrm{e}}$ siècle av. J.-C.

44. Coulson (1985, 1986 et 1988).

45. Cartledge (1992) ; Eder (1998, 107-09) ; Kõiv (2003, 63-66) ; Zavvou (2009, 29-31, fig. 4.7).

46. Vlachou $(2015,116)$.

47. Encore récemment on croyait que Sparte avait été fondée autour de $950 \mathrm{av}$. J.-C., après une rupture d'occupation dans la vallée de l'Eurotas entre 1050 et 950 av. J.-C.

48. Tosti (2017).

49. Lupi $(2008,369)$.

50. Des traces de cette mémoire d'événements dits historiques sont conservées dans la documentation littéraire sous le nom de la tradition autour du "retour des Héraclides". Par exemple, la célébration annuelle des Karneia en garde certains éléments : cf. Thucydide, V, 54, 2 ; Pausanias, III, 13, 3-4. 
51. Lupi $(2017,45)$.

52. Tyrtée fr. 2 West ; Lupi $(2017,46)$.

53. Lupi $(2017,47)$.

54. Dans la même aire funéraire, deux autres tombes d'époques plus récentes ont été creusées, témoignant ainsi d'une continuité dans l'utilisation des espaces. Voir Zavvou (1997, 160-163, pl. 2, fig. 71), Raftopoulou (1997, 272), Raftopoulou (1998, 133, fig. 12.14), Raftopoulou (2001), ThemosZavvou (2010, 228-229).

55. Raftopoulou (1998, 133, fig. 12.15).

56. Flouris $(1996,123-125$, pl. 11, fig. 44 b) ; Zavvou-Themos $(2009,116)$; Tosti $(2011)$. La tombe de la période géométrique, couverte à son tour par une couche de terre mélangée à des fragments de céramique géométrique, conservait le défunt en position accroupie, avec un anneau en bronze à un doigt. Elle faisait probablement partie d'une nécropole géométrique.

57. On distingue des fragments de canthares décorés de files de losanges ou de lignes brisées; des fragments de coupes; une partie d'un cotyle tardo-géométrique décoré au-dessous du rebord d'une frise continue qui court le long de toute la circonférence, avec à l'intérieur la représentation stylisée d'une file d'oiseaux, tandis que le corps du vase est caractérisé par deux métopes plus petites, dont l'intérieur est enrichi d'un motif en zigzag; une partie d'une lékané avec une décoration à triple ligne ondulée dans la métope centrale; un petit lécythe fragmentaire de la période orientalisante ; des canthares miniatures.

58. Spyropoulos (1981a, 121, pl. 1, fig. 58 a-d).

59. Raftopoulou (1998, 133, fig. 12.16).

60. Steinhauer (1972, 242-246, fig. 1, pl. 178 a-6, 179 a-e, 180 a-6, 181 a-6, 182 a-d, 183 a) ; Steinhauer (1973-1974, 286).

61. Efstathiou $(1997,190)$; Efstathiou (1999, 174, pl. 20, fig. 21). Cf. Zavvou \& Themos $(2009,111)$.

62. Steinhauer $(1972,245)$. Le rituel funéraire et l'absence de mobilier laisseraient penser à une date contemporaine aux tombes de la période géométrique retrouvées en odos Staufert 5.

63. Steinhauer $(1972,244)$. La tombe ressemble à celles découvertes à l'est et au sud de l'Acropole. 64. Zavvou (1996, 29-131, fig. 45 a-b) ; Zavvou (1998, 172-173); Raftopoulou (1997, 278).

65. Les fondations du mur du péribole du sanctuaire coupent une couche de terre noire riche en fragments de céramique de l'époque géométrique. La même couche se retrouve au sud du mur et dans toute la zone environnante, comme les fouilles de l'École britannique d'Athènes l'ont démontré : cf. Dickins (1906-1907, 143-144), Woodward-Hobling (1923-1925, 246, 249-252) et Woodward-Hobling (1926-1927, 38). De la même phase chronologique date un mur de $5 \mathrm{~m}$ de long, construit en pierres sèches, orienté est/ouest. Ce petit mur est identifié comme le premier autel du sanctuaire. Voir Dickins (1906-1907, 144-146); voir aussi Spallino (2016), avec bibliographie précédente.

66. Dawkins et al. (1929).

67. Vlizos $(2015,97)$. À propos de la translation du culte du héros local de l'époque mycénienne Hyakynthos au culte "dorien» d'Apollon Amyklaios, voir Calligas (1992, 46); voir aussi Georgoulaki (1994) et Petropoulou (2015), avec bibliographie précédente.

68. De Polignac $(1995,1996,2005)$.

69. Voir Lupi (2018).

70. La Grande Rhètra, mentionnée par Plutarque (Lycurgue, 6, 1-10), est considérée comme le fondement constitutionnel de l'État spartiate. Pour la chronologie, voir Pettersson (1992, 112-116). Sur l'institutionnalisation politique de Sparte, voir Nafissi (2009, 127), Finley (1968), Hodkinson (2000, 3-4), Lupi (2005, 210-213), Lupi (2008, 371-374) et van Wees (1999).

71. Greco $(1994,593)$. 


\title{
RÉSUMÉS
}

Les évolutions institutionnelles et les modes d'occupation du territoire qui ont amené à la création de la polis de Sparte dans la vallée du fleuve Eurotas entre les $\mathrm{X}^{\mathrm{e}}$ et IX $\mathrm{X}^{\mathrm{e}}$ siècles av. J.-C. sont encore aujourd'hui peu claires. La lecture assez superficielle des textes a donné lieu à la création du modèle que les dynamiques de peuplement définissent comme kata kômas. D'autre part, les sources archéologiques, peu nombreuses et sans cohérence apparente, sont difficilement exploitables et à première vue ne permettent pas de s'écarter des modèles d'interprétation qui expliquent la naissance de Sparte comme le résultat de ses institutions politiques. La référence majeure est toujours la figure légendaire de Lycurgue, ainsi que l'arrivée des Doriens dans le Péloponnèse. Avec la présente étude, nous proposons une nouvelle lecture des données disponibles, qui fait davantage dialoguer celles-ci et dévoilera le processus d'idéalisation - le mirage spartiate - qui se développa au fur et à mesure de la consolidation de la cité laconienne.

The institutional development and the modes of occupation of the territory which led to the creation of the polis of Sparta in the valley of the Eurotas river between the 10th and 9th centuries $\mathrm{BC}$ are still unclear today. The rather superficial reading of the texts gave rise to the creation of a model that the dynamics of settlement define as kata kômas. On the other hand, the archaeological evidence, few in number and without apparent coherence, are difficult to exploit and, at first glance, do not allow us to shift from the models of interpretation which explain the birth of Sparta as the result of its own political institutions. The major reference is still the legendary figure of Lycurgus, as well as the arrival of the Dorians in the Peloponnese. In the present paper, I propose a new assessment of the available data, reinforcing their dialogue and revealing the idealization process-the Spartan mirage-which developed as the Laconian city was consolidated.

\section{INDEX}

Mots-clés : naissance de la polis, Sparte, urbanisation, topographie, âge du fer, kata kômas

Keywords : formation of the polis, Sparta, urbanization, topography, Iron Age, kata kômas

\author{
AUTEUR \\ VALERIA TOSTI \\ Université Paris 1 Panthéon-Sorbonne \\ valeria.tosti@univ-paris1.fr
}

